\title{
Current and Future Roles of Targeted Therapy and Immunotherapy in Advanced Melanoma
}

\author{
Anthony J. Olszanski, MD, RPh
}

\begin{abstract}
BACKGROUND: Melanoma is an aggressive disease that accounts for approximately $75 \%$ of skin cancer-related deaths. Historically, treatment options for patients with advanced stage melanoma have been limited by modest response rates and failure to improve overall survival. The treatment landscape for advanced stage melanoma was revolutionized in 2011 with the approval of ipilimumab and vemurafenib, both of which improved overall survival in phase III clinical trials. More recently, the targeted inhibitors dabrafenib and trametinib have demonstrated similar therapeutic profiles.
\end{abstract}

OBJECTIVES: To (a) discuss emerging treatment options for advanced melanoma, specifically ilpilimumab, vemurafenib, dabrafenib, and trametinib, in the context of their mechanisms of action and their potential for longterm improvement in patient outcome, and (b) to consider the impact of these agents on the current treatment landscape.

METHODS: A literature search was conducted to collect data from clinical trials involving ipilimumab, vemurafenib, dabrafenib, and trametinib. Emphasis was placed on outcome measures related to long-term clinical benefit.

RESULTS: Ipilimumab, a fully human monoclonal antibody, exploits the natural ability of the immune system to eradicate primary cancer cells. It inhibits the binding of cytotoxic T-lymphocyte antigen-4 to its ligands, thereby potentiating T-cell response and antitumor immunity. In a phase III clinical trial, ipilimumab at $3 \mathrm{mg} / \mathrm{kg}$ improved overall survival in previously treated patients with metastatic melanoma. Components of the mitogenactivated protein kinase (MAPK) pathway are particularly relevant in melanoma and have been targeted by small molecular inhibitors. Vemurafenib and dabrafenib inhibit the BRAF V600 mutation, which prevents oncogenic activities such as uncheck proliferation and evasion of immune response. Data from phase III clinical trials suggest that both vemurafenib and dabrafenib improve patient outcomes, with vemurafenib showing an overall survival benefit and dabrafenib showing improved median progression-free survival. The targeted-therapy approach in melanoma continued to gain momentum with the development of trametinib, which inhibits the MEK protein, the only known substrate of the BRAF V600 protein. Inhibition of MEK leads to decreased cell signaling and proliferation in cancer cells. In phase III trials, trametinib demonstrated significant improvement in median progression-free survival and median overall survival compared with chemotherapy treatment, making this treatment a valuable addition to the current armamentarium. The adverse events associated with these new treatments are generally tolerable and mild to moderate in severity; however, care should be taken when selecting a therapy, since the specific adverse events associated with these treatments are unique, and serious events have been reported.

CONCLUSIONS: The immunotherapy ipilimumab and the MAPK-targeted inhibitors vemurafenib, dabrafenib, and trametinib have forever changed the treatment landscape for melanoma. Indeed, these new therapies have demonstrated long-term improvement in patient outcome, a benefit not afforded by traditional therapeutics. Important research continues on the molecular basis of melanoma, and new targets are likely to emerge. Other areas of work include optimization of sequencing and/or combination of current treatments, which may increase the number of patients who experience clinical benefit.

J Manag Care Pharm. 2014;20(4):346-56

Copyright $\odot 2014$, Academy of Managed Care Pharmacy. All rights reserved.

\section{What is already known about this subject}

- Melanoma accounts for less than 5\% of cases of skin cancer; however, it accounts for most (75\%) skin cancer-related deaths.

- Systemic chemotherapeutic options for metastatic melanoma have little benefit, without improvements in overall survival.

- Up to $50 \%$ of patients with metastatic melanoma harbor an activating mutation of the BRAF gene.

\section{What this study adds}

- Immunotherapy using the cytotoxic T-lymphocyte antigen-4 inhibitor ipilimumab was the first drug approved by the FDA to show a survival advantage.

- Three mutation-directed targeted agents, vemurafenib, dabrafenib, and trametinib, have been approved by the FDA and offer a survival advantage in metastatic melanoma. Vemurafenib and dabrafenib are BRAF inhibitors while trametinib is an MEK inhibitor.

- Trametinib is not indicated for patients who have previously progressed on a BRAF inhibitor.

- New immunotherapeutic options, such as inhibitors of the PD-1 and PD-L1 proteins, have shown substantial promise to date and may significantly impact the treatment and outcome of patients with metastatic melanoma in the near future.

S kin cancer is the most common form of cancer in the United States. ${ }^{1}$ Most patients develop nonmelanoma skin cancer, where surgical excision of the primary lesion remains effective for early disease, and survival rate is high. Although melanoma accounts for less than $5 \%$ of cases of skin cancer, it accounts for most (75\%) skin cancer-related deaths. It is estimated that, in 2014 , almost 76,100 men and women in the United States will be diagnosed with invasive melanoma of the skin, and close to 9,710 people will die from the disease. Furthermore, melanoma is the most common form of cancer for young adults aged 25 to 29 years and the second most common form of cancer for young people aged 15 to 29 years. $^{2}$ Given the prevalence of melanoma in young individuals, it accounts for 1 of the 10 leading causes of years of life lost. ${ }^{3}$ The overall 5 -year survival rate from diagnosis for patients whose melanoma is detected early, before the tumor has spread to regional lymph nodes or other organs, is about $98 \%$ in the United States, falling to $62 \%$ when the disease reaches 


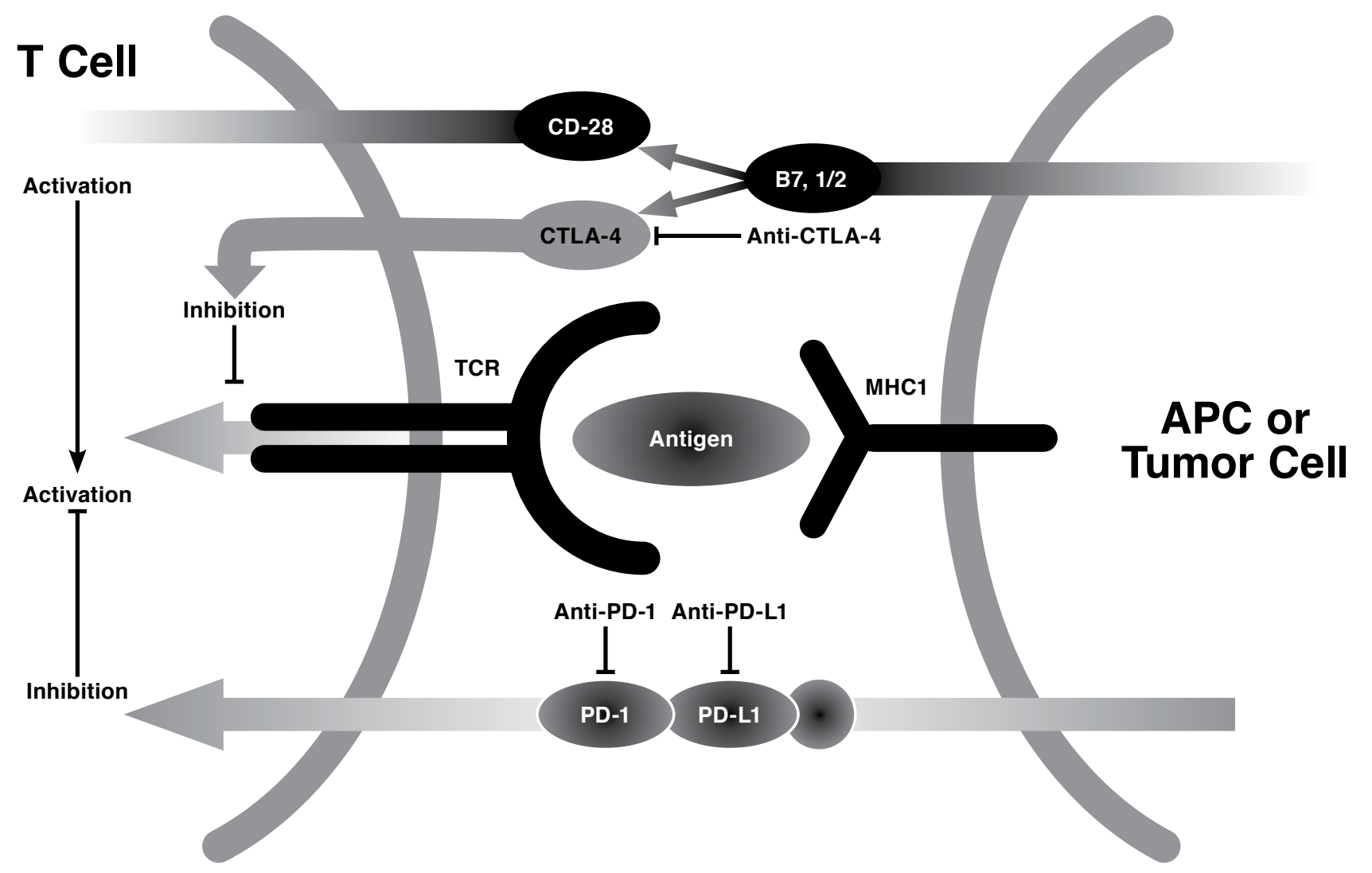

the lymph nodes, and 15\% when the disease metastasizes to distant organs. ${ }^{4}$ In a meta-analysis of patients with advanced, metastatic melanoma enrolled in phase II clinical trials, the 1 -year survival rate from clinical trial enrollment was approximately 25\%, and median overall survival (OS) was only 6 months. ${ }^{5}$

Until recently, systemic chemotherapies (dacarbazine [DTIC]), hydroxyurea, or immunotherapy with high-dose interleukin-2 (IL-2) were the only treatment options approved by the U.S. Food and Drug Administration (FDA) for patients with advanced melanoma. ${ }^{6-8}$ These agents have not demonstrated improvement in OS, nor have they been studied in large randomized phase III trials. Therefore, new strategies are continually sought to improve patients' quantity of life, as measured by OS, and to improve their quality of life with the ultimate goal of living a healthier, longer, and productive life.

After decades of clinical research that has failed to improve survival for patients with advanced melanoma, the treatment landscape changed dramatically in 2011 with the FDA approval of 2 new agents, ipilimumab and vemurafenib, on the basis of significantly improved OS in randomized phase III tri- als of patients with advanced melanoma. ${ }^{9-11}$ Both agents yielded incremental increases in OS that were never before realized in patients with advanced melanoma. However, the cost of each of these novel biologic treatments is substantially higher than that associated with previously available therapies for this population. When surveyed, payers indicated a minimum improvement of 6 months in median OS would be necessary to define treatment as "good value."12 Oncologists presented with hypothetical scenarios deemed therapies with an average of approximately $\$ 300,000$ per quality-adjusted-life-year as "good value," although $75 \%$ stated that patients should have access to effective care regardless of cost. ${ }^{13}$

In this article, we discuss current treatments for advanced melanoma and consider their value in terms of clinical benefit to the patient and the potential for providing long-term improvements in patient outcomes.

\section{Understanding the Current Armamentarium}

\section{Anti-CTLA-4 Antibodies}

Ipilimumab is an antibody against cytotoxic T-lymphocyte antigen-4 (CTLA-4) that was approved by the FDA in 2011 
Current and Future Roles of Targeted Therapy and Immunotherapy in Advanced Melanoma

\section{TABLE 1 Overall Survival Rates with Ipilimumab in Phase II and III Studies}

\begin{tabular}{|c|c|c|c|c|c|c|c|c|}
\hline \multirow[b]{2}{*}{ Study } & \multicolumn{8}{|c|}{ Survival Rate, $\%(95 \% \text { CI })^{a}$} \\
\hline & & 1 Year & & 2 Year & & 3 Year & & 4 Year \\
\hline \multicolumn{9}{|l|}{ MDX010-20b $(\mathrm{N}=676)^{9}$} \\
\hline $3 \mathrm{mg} / \mathrm{kg}+\mathrm{gpl00}$, previously treated $(\mathrm{n}=403)$ & & 43.6 & & 21.6 & & N/R & & $\mathrm{N} / \mathrm{R}$ \\
\hline $3 \mathrm{mg} / \mathrm{kg}$, previously treated $(\mathrm{n}=137)$ & & 45.6 & & 23.5 & & N/R & & $\mathrm{N} / \mathrm{R}$ \\
\hline gpl00, previously treated $(\mathrm{n}=136)$ & & 25.3 & & 13.7 & & $\mathrm{~N} / \mathrm{R}$ & & $\mathrm{N} / \mathrm{R}$ \\
\hline \multicolumn{9}{|l|}{ CA184-024b $(\mathrm{N}=502)^{10}$} \\
\hline 10 mg/kg + DTIC, treatment-naïve $(\mathrm{n}=250)$ & & 47.3 & & 28.5 & & 20.8 & & $\mathrm{~N} / \mathrm{R}$ \\
\hline DTIC, treatment naïve $(\mathrm{n}=252)$ & & 36.3 & & 17.9 & & 12.2 & & $\mathrm{~N} / \mathrm{R}$ \\
\hline \multicolumn{9}{|l|}{ CA184-008 $(\mathrm{N}=155)^{16}$} \\
\hline $10 \mathrm{mg} / \mathrm{kg}$, previously treated & 47.2 & $(39.5-55.1)$ & 32.8 & $(25.4-40.5)$ & 23.3 & $(16.7-30.4)$ & 19.7 & $(13.4-26.5)$ \\
\hline \multicolumn{9}{|l|}{ CA184-022 $(\mathrm{N}=217)^{16}$} \\
\hline $10 \mathrm{mg} / \mathrm{kg}$, previously treated $(\mathrm{n}=72)$ & 48.6 & $(36.8-60.4)$ & 29.8 & $(19.1-41.1)$ & 24.8 & $(14.8-35.7)$ & 21.5 & $(11.9-32.0)$ \\
\hline $3 \mathrm{mg} / \mathrm{kg}$, previously treated $(\mathrm{n}=72)^{\mathrm{c}}$ & 39.3 & $(28.0-50.9)$ & 24.2 & $(14.4-34.8)$ & 19.7 & $(10.7-29.4)$ & 18.2 & $(9.5-27.6)$ \\
\hline $0.3 \mathrm{mg} / \mathrm{kg}$, previously treated $(\mathrm{n}=73)^{\mathrm{c}}$ & 39.6 & $(28.2-51.2)$ & 18.4 & $(9.6-28.2)$ & 13.8 & $(6.1-22.5)$ & 13.8 & $(6.1-22.5)$ \\
\hline \multicolumn{9}{|l|}{ CA184-007 $(\mathrm{N}=115)^{16}$} \\
\hline Ipilimumab + placebo $(\mathrm{n}=57)$ & 62.4 & $(49.4-75.1)$ & 41.8 & $(28.3-55.5)$ & 34.4 & $(21.1-48.2)$ & 32.0 & $(18.9-45.7)$ \\
\hline $10 \mathrm{mg} / \mathrm{kg}$, treatment-naïve $(\mathrm{n}=32)$ & 71.4 & $(55.2-87.2)$ & 56.6 & $(38.4-74.3)$ & 42.5 & $(23.0-62.0)$ & 37.7 & $(18.6-57.4)$ \\
\hline $10 \mathrm{mg} / \mathrm{kg}$, previously treated $(\mathrm{n}=25)$ & 50.8 & $(31.5-71.1)$ & 24.2 & $(8.0-42.8)$ & 24.2 & $(8.0-42.8)$ & 24.2 & $(8.0-42.8)$ \\
\hline Ipilimumab + budesonide $(n=58)$ & 55.9 & $(42.7-68.8)$ & 41.1 & $(27.7-54.8)$ & 38.7 & $(25.2-52.4)$ & 36.2 & $(22.9-49.9)$ \\
\hline $10 \mathrm{mg} / \mathrm{kg}$, treatment-naïve $(\mathrm{n}=21)$ & 65.9 & $(45.0-85.7)$ & 57.7 & $(33.3-81.0)$ & 57.7 & $(33.3-81.0)$ & 49.5 & $(23.8-75.4)$ \\
\hline $10 \mathrm{mg} / \mathrm{kg}$, previously treated $(\mathrm{n}=37)$ & 49.9 & $(33.3-66.6)$ & 31.6 & $(16.5-47.6)$ & 28.4 & $(13.9-44.2)$ & 28.4 & $(13.9-44.2)$ \\
\hline \multicolumn{9}{|c|}{$\begin{array}{l}\text { a Based on Kaplan-Meier estimation with CIs computed using the bootstrap method; analyses include all randomized patients for studies CA184-007 and CA184-022 and } \\
\text { all treated patients for CA184-008. } \\
{ }^{b} \mathrm{CI} \text { not available for MDX010-20 and CA184-024. } \\
{ }^{c} \text { In the } 0.3 \text { and } 3 \mathrm{mg} / \mathrm{kg} \text { dose groups, } 33 \% \text { and } 42 \% \text { of patients, respectively, crossed over to the } 10 \mathrm{mg} / \mathrm{kg} \text { dose group. } \\
C I=\text { confidence interval; DTIC= dacarbazine; } \mathrm{mg} / \mathrm{kg}=\text { milligram per kilogram; NR=not reported. }\end{array}$} \\
\hline
\end{tabular}

for adult patients with unresectable or metastatic melanoma. ${ }^{14}$ CTLA-4 acts to downregulate T-cell activity, controlling both the duration and intensity of immune responses and preventing unwanted autoimmunity against self-antigens by inducing immune tolerance..$^{15}$ Ipilimumab blocks CTLA-4, thereby allowing appropriate T-cell activation through re-establishment of the costimulatory binding of CD28 to B7 via the critical interaction between the $\mathrm{T}$ cell and antigen presenting cell. This interaction restores $\mathrm{T}$-cell proliferation and amplifies T-cellmediated immunity, thus, enhancing the patient's capacity to mount an antitumor immune response (Figure 1).

The efficacy of ipilimumab has been investigated in 2 large phase III trials of patients with advanced melanoma: at the approved dose of 3 milligrams per kilograms $(\mathrm{mg} / \mathrm{kg})$ in previously treated patients and at $10 \mathrm{mg} / \mathrm{kg}$ in combination with DTIC in treatment-naive patients (Table 1). ${ }^{9,10}$ In the treatmentnaïve population, median $O S$ with ipilimumab $10 \mathrm{mg} / \mathrm{kg}$ plus DTIC was 11.2 months, with a $28 \%$ reduction in the risk of death compared with DTIC monotherapy (9.1 months, hazard ratio $[\mathrm{HR}]=0.72, P<0.001)$. In the previously treated patients, median OS with ipilimumab $3 \mathrm{mg} / \mathrm{kg}$ monotherapy was 10.1 months, and the risk of death was reduced by $34 \%$ compared with the gp100 vaccine comparator ( 6.4 months, $\mathrm{HR}=0.66, P=0.003$ ).
CTLA-4 expression on T cells is ubiquitous. Thus, the activity of ipilimumab is not dependent on the genetic make-up of the tumor or the presence of specific mutations; therefore, in theory, it has the potential to be efficacious in all tumors in a patient. ${ }^{16,17}$ The phase III studies support this hypothesis: the effect of ipilimumab on OS was observed independent of age, sex, baseline serum lactate dehydrogenase (LDH) levels, stage of metastatic disease, and previous IL-2 therapy. Importantly, survival curves separated and plateaued, suggesting that a proportion of patients experienced prolonged disease control, with some patients surviving in excess of 4 years. ${ }^{16}$ In a landmark analysis of 177 patients previously treated with ipilimumab in phase II clinical trials at the National Cancer Institute, 13\% to $25 \%$ of patients survived at least 5 years; survival curves generally leveled off for patients surviving beyond 4 years, revealing a notable durability of the immune response in appropriate patients. ${ }^{18}$ In a second study of extended treatment or follow-up in patients previously enrolled in 1 of 3 different phase II trials, 5-year survival rates ranged from $12.3 \%$ to $>49 \%$ among both treatment-naive and previously treated patients treated with ipilimumab $10 \mathrm{mg} / \mathrm{kg}$, and importantly, a meaningful proportion of patients continued to survive beyond 5 years (Table 1). ${ }^{19}$ This durability of response is an important 
Current and Future Roles of Targeted Therapy and Immunotherapy in Advanced Melanoma

TABLE 2 Key Results from Selective BRAF and MEK Inhibitor Studies

\begin{tabular}{|c|c|c|c|c|c|}
\hline Study & Agent & Phase & Study Arms & Median PFS (Months) & Median OS (Months) \\
\hline \multirow[t]{2}{*}{ Chapman et al. ${ }^{23}$} & \multirow[t]{2}{*}{ Vemurafenib } & \multirow[t]{2}{*}{ III } & $\begin{array}{l}\text { Vemurafenib } 960 \mathrm{mg} \\
\text { PO BID }\end{array}$ & 6.9 & 13.6 \\
\hline & & & $\begin{array}{c}\text { DTIC } \\
1,000 \mathrm{mg} / \mathrm{m}^{2} \\
\text { IV every } 3 \text { weeks }\end{array}$ & $\begin{array}{c}1.6(\mathrm{HR}=0.38 \\
95 \% \mathrm{CI}=0.32-0.46 ; P<0.001)\end{array}$ & $\begin{array}{c}9.7 \text { (HR 0.07; } \\
95 \% \text { CI 0.57-0.87; } \\
\text { P<0.001) }\end{array}$ \\
\hline \multirow[t]{2}{*}{ Flaherty et al. ${ }^{27}$} & \multirow[t]{2}{*}{$\begin{array}{l}\text { Dabrafenib, } \\
\text { trametinib }\end{array}$} & \multirow[t]{2}{*}{ I/II } & $\begin{array}{l}\text { Dabrafenib ( } 75 \text { or } 150 \mathrm{mg} \text { PO BID) and } \\
\text { trametinib (1, } 1.5 \text {, or } 2 \mathrm{mg} \text { PO BID) }\end{array}$ & 9.4 & NR \\
\hline & & & Dabrafenib monotherapy & $\begin{array}{c}5.8(\mathrm{HR}=0.39 \\
95 \% \mathrm{CI}=0.25-0.62 ; P<0.001)\end{array}$ & NR \\
\hline Falchook et al. ${ }^{32}$ & Dabrafenib & I/II & $\begin{array}{c}\text { Dabrafenib dose } \\
\text { escalation to } 300 \mathrm{mg} \\
\text { PO BID }\end{array}$ & $\begin{array}{c}5.5 \\
(95 \% \mathrm{CI}=4.1-8.3)\end{array}$ & NR \\
\hline
\end{tabular}

$B I D=$ twice a day; $C I=$ confidence interval; $D T I C=$ dacarbazine; $H R=$ hazard ratio; $I V=$ intravenous; $m g / m^{2}=$ milligram per square meter; NR=not reported; OS = overall survival; $\mathrm{PFS}=$ progression-free survival; $\mathrm{PO}=$ orally

differentiating feature when considering first-line treatment with the checkpoint inhibitors/immunomodulators compared with shorter duration of response seen with the kinase inhibitors in patients who are BRAF mutation-positive.

Presumably these extended responses are due to the action mechanism of ipilimumab, which is immunologic in nature. Researchers have also observed that antitumor immune responses can develop over weeks to months, and in some patients, apparent initial "progression" has been followed by a slow and steady decline in tumor burden or stable disease..$^{20,21}$ Therefore, the assessment of benefit at an early time point during or after completion of the prescribed 4 doses must take into account not only the imaging results, but also the patient's general well-being and laboratory parameters such as the LDH levels.

\section{Signal Transduction Small Molecule Inhibitors}

Vemurafenib and dabrafenib are oral enzyme inhibitors of the oncogenic BRAF V600 protein kinase. Vemurafenib was approved by the FDA in 2011, and dabrafenib was approved in 2013 for the treatment of adult patients with BRAF V600 mutation-positive unresectable or metastatic melanoma. ${ }^{22}$ $\mathrm{BRAF}$ is a serine/threonine protein kinase that activates the mitogen-activated protein kinase (MAPK) signaling pathway. Approximately $40 \%$ to $50 \%$ of melanomas harbor activating BRAF mutations, more than $90 \%$ of which are at codon 600 and comprise a single-nucleotide mutation resulting in substitution of glutamic acid for valine (BRAF V600E). By inhibiting the BRAF V600E mutation, vemurafenib acts to oppose its oncogenic activities, such as evasion of senescence and apoptosis, unchecked proliferation, angiogenesis, tissue invasion and metastasis, and evasion of immune response.

Vemurafenib (960 mg twice daily) received FDA approval based on interim 6-month results from a phase III trial for previously untreated patients with BRAF V600E mutation-positive metastatic melanoma. ${ }^{11}$ With 12.5 months follow-up and cen- soring of patients who crossed over from DTIC to vemurafenib, median OS with vemurafenib was reported to be 13.6 months versus 9.6 in the comparator arm; the risk of death compared with DTIC was 30\% $(P<0.001 ; 24 \%$ without censoring; Table 2). ${ }^{23}$ Notably, in a post hoc analysis, the benefit of vemurafenib relative to DTIC was greatest in patients with stage Mlc disease and increased LDH concentration. Many patients treated with vemurafenib had a rapid tumor response with resultant decreased tumor burden as indicated by the high response rate. Unfortunately, most patients progressed within about 6 months, presumably because of acquired resistance through various mechanisms. Research indicates that resistance may be acquired from the MAPK pathway-dependent and MAPK pathway-independent mechanisms that allow tumors in patients with melanoma to survive despite continued BRAF inhibition..$^{24-26}$ To overcome the impact of resistance to vemurafenib, the combination of BRAF and MEK inhibitors is being investigated in clinical trials, with initial data indicating that the combination therapy may provide a more durable response rate and may prolong progression-free survival (PFS) compared with either agent alone. ${ }^{27-30}$ Interestingly, another strategy being investigated in clinical trials that may help overcome BRAF inhibition resistance is the use of intermittent dosing of vemurafenib. ${ }^{31}$

Dabrafenib is another BRAF inhibitor that has demonstrated efficacy in patients with BRAF-mutated metastatic melanoma (Table 2). The phase I study of dabrafenib involved 184 patients, including 156 with metastatic melanoma. ${ }^{32}$ Dabrafenib treatment at the phase II recommended dose (150 mg twice daily) resulted in complete or partial response in 25 of 36 patients (69\%) with BRAF V600 mutation. Antitumor activity was durable, with 17 patients (47\%) on treatment for approximately 6 months. In the phase II study, 92 treatmentnaive patients with melanoma were treated with dabrafenib at the phase II recommended dose. ${ }^{33}$ Response rates were affected by BRAF V600 mutation status; patients with the BRAF V600E 


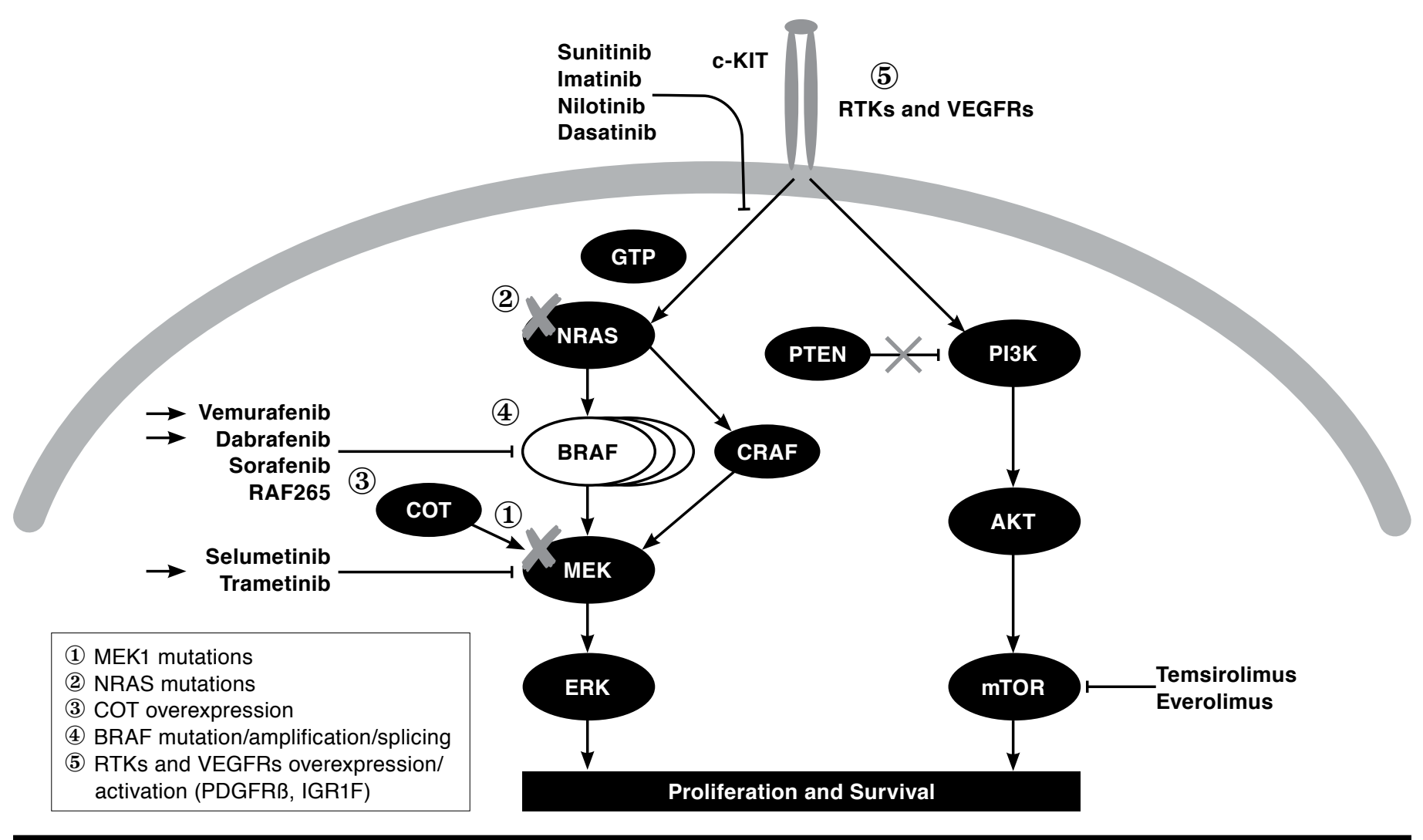

mutation showed a $60 \%$ response rate $(53 \%$ partial, $7 \%$ complete), whereas patients with the less frequently observed BRAF V600K mutation showed only partial responses (13\%). These findings highlight the importance of screening for BRAF V600 mutation status in order to identify patients who are most likely to derive the greatest benefit from dabrafenib treatment. The phase III study of dabrafenib was designed to compare the activity of dabrafenib with DTIC. ${ }^{29}$ Patients with BRAF-mutant metastatic melanoma were randomly assigned to receive either dabrafenib (187 patients; dosage $150 \mathrm{mg}$ twice daily) or DTIC (63 patients; dosage 1,000 milligrams per square meter [ $\left.\mathrm{mg} / \mathrm{m}^{2}\right]$ every 3 weeks). Response rate was significantly higher in the dabrafenib group (50\%) than in the DTIC group (6\%). Similarly, median PFS (mPFS) was longer in the dabrafenib group (5.1 months) than in the DTIC group (2.7 months; $P<0.0001)$. Taken together, these data suggest that dabrafenib demonstrates significant improvement in response rate and $\mathrm{mPFS}$ compared with DTIC in BRAF-mutant metastatic melanoma.

Trametinib is another enzyme inhibitor that is an effective therapeutic for the treatment of metastatic melanoma. This treatment option is similar to vemurafenib and dabrafenib in that it also targets the MAPK pathway (Figure 2). ${ }^{34}$ Specifically, however, trametinib acts by inhibiting MEK, the only known substrate of BRAF, which in turn leads to decreased cell signaling and proliferation in cancer cells. Trametinib (2 mg orally once daily) was FDA-approved in 2013 for the treatment of adult patients with the BRAF V600E/K mutation and unresectable or metastatic melanoma. ${ }^{35}$ A phase II trial of trametinib enrolled 97 patients with BRAF-mutant melanoma and divided patients into 2 cohorts. Patients previously treated with BRAF inhibitor (cohort $A, n=40$ ) showed no confirmed objective response, whereas those previously treated with chemotherapy and/or immunotherapy (cohort $\mathrm{B}, \mathrm{n}=57$ ) showed a 25\% response rate, suggesting that $\mathrm{BRAF-inhibitor} \mathrm{resistance} \mathrm{develops} \mathrm{with}$ repeated exposure. It is important to note that trametinib, therefore, is not indicated in patients who have received prior BRAF-inhibitor therapy. In a recent phase III trial, the activity of trametinib was compared with chemotherapy (DTIC or paclitaxel) in 322 patients with BRAF-mutated melanoma in the first-line setting. Median PFS and OS were greater in the trametinib group ( $\mathrm{mPFS}=4.8$ months; $\mathrm{OS}=81 \%$ ) than in the chemotherapy group (mPFS = 1.5 months; OS =67\%). ${ }^{36}$ 


\section{Safety of Ipilimumab and Enzyme Inhibitors}

Given that ipilimumab and enzyme inhibitors have different mechanisms of action, it is not surprising that these treatments also have different adverse event (AE) profiles. Ipilimumab is associated with inflammatory adverse reactions resulting from increased or excessive immune activity, which can be severe or life threatening and most commonly affect the gastrointestinal, liver, skin, nervous, and endocrine systems. ${ }^{37}$ These immune-related AEs (irAEs) have been seen consistently across clinical studies and are generally manageable and reversible without long-term consequences if recognized early and treated promptly. ${ }^{37}$ Most patients receiving ipilimumab have mild to moderate toxicity. In the phase III second-line trial reported by Hodi et al. (2010), ${ }^{9} 80$ out of 131 patients (61\%) treated with ipilimumab $3 \mathrm{mg} / \mathrm{kg}$ had an irAE, the majority of which were grade 1 or 2 . Approximately $15 \%$ of patients $(n=19)$ experienced a grade 3 or 4 toxicity. ${ }^{9}$ The irAEs demonstrated a consistent profile throughout the clinical development program. Therefore, ipilimumab and treatment can be closely managed in the community setting through the use of readily available algorithms that present detailed guidance on the management of particular AEs according to their severity. ${ }^{38}$ Using these algorithms, which rely on prompt recognition, intervention, and the use of oral and/or parenteral corticosteroids, most irAEs are effectively managed and resolve within 6 to 8 weeks of onset. ${ }^{39}$

The safety profiles for vemurafenib and dabrafenib are similar; however, vemurafenib was typically associated with photosensitivity whereas dabrafenib was mainly associated with pyrexia. ${ }^{22,33}$ These events were generally manageable with appropriate clinical care and effective patient education. Approximately a quarter to one-third of patients in vemurafenib clinical trials developed squamous cell carcinoma of the skin, the majority of which were keratoacanthoma type. ${ }^{11}$ Early recognition through dermatologic exams and referral to a dermatologist is paramount.

Given the high response rates noted in patients with a V600 mutation treated with vemurafenib and dabrafenib, and the long-term durability of response noted in patients treated with ipilimumab, combination strategies have also been pursued. A recent pooled analysis of 1,861 patients treated with ipilimumab across clinical development of the drug showed that the median OS was 11.4 months, and among these patients, $254(22 \%)$ were still alive after 3 years. A plateau in survival of the patients, starting at 3 years and continuing through to 10 years, appears to be independent of dose ( 3 or $10 \mathrm{mg} / \mathrm{kg}$ ), whether the patients had received previous treatment or not, and whether or not they had been kept on a maintenance dose of the drug. ${ }^{40}$ Unfortunately, as describe by Ribas et al. (2013), the combination of vemurafenib plus ipilimumab led to significant hepatotoxicity. ${ }^{41}$ Ipilimumab and vemurafenib should not be combined outside of a clinical trial setting, given this finding, until combination strategies are further pursued in the context of a clinical trial setting.

Overall, the MEK inhibitor trametinib is well tolerated. The most frequently reported side effects were cutaneous AEs, including rash, which occurred in $75 \%$ to $90 \%$ of patients. ${ }^{28,30,42}$ Gastrointestinal events including diarrhea, nausea, and vomiting have also been reported with trametinib treatment; however, these AEs were generally responsive to standard supportive care measures. Additional AEs, including dry eye and blurred or impaired vision, resolve upon treatment discontinuation.

\section{Ipilimumab Retreatment}

Clinical studies have looked at the question of retreating patients with ipilimumab; the other agents discussed here have yet to publish retreatment data. Ipilimumab is administered as a 90-minute intravenous infusion at a dose of $3 \mathrm{mg} / \mathrm{kg}$ every 3 weeks for 4 doses over a period of 12 weeks (induction). Extended follow-up of melanoma patients indicates that the effects of ipilimumab are persistent, even in the absence of continued treatment. However, durable immune responses are also capable of altering the phenotype of the tumor, which may result in impaired or decreased response over time. ${ }^{43}$ Emerging evidence suggests that restarting ipilimumab after disease progression may reactivate the primed immune system to recognize and respond to any remaining tumor cells or new tumor cells that have appeared. ${ }^{43}$ The National Comprehensive Cancer Network (NCCN) guidelines state that retreatment with ipilimumab may be considered for select patients who experience no significant systemic toxicity during their initial treatment and who relapse after an initial clinical response (complete or partial response) to ipilimumab or stable disease lasting at least 3 months. ${ }^{44}$ In the Hodi et al. phase III trial, 31 patients were retreated with ipilimumab (alone or in combination with gp100). Of these, 6 patients (19\%) had a durable complete or partial response upon retreatment, with 15 patients regaining disease stabilization, for a disease control rate of $68 \%$. The frequency and type of AEs observed during retreatment were similar to those observed during the initial dosing regimen, and toxicities observed during induction did not appear to predispose patients to retreatment toxicity. ${ }^{43}$ Although patient numbers were too small to draw definitive conclusions, retreatment with ipilimumab in patients who meet defined criteria appears to be a feasible intervention. The concept of reactivating an immune response with ipilimumab through retreatment is currently being evaluated in a prospective phase II trial (NCT01709162). ${ }^{43}$ 


\section{Use of Therapy in Specific Subpopulations}

Ipilimumab at $3 \mathrm{mg} / \mathrm{kg}$ is approved for use in patients with unresectable or metastatic melanoma, irrespective of melanoma subtype. Ninety percent of diagnosed melanomas are superficial spreading melanoma, nodular melanoma, or lentigno malignant melanomas. ${ }^{45}$ The remaining $10 \%$ are composed of acral lentiginous melanoma (most commonly found on the palms of the hands and soles of the feet) and noncutaneous melanomas such as ocular and mucosal melanoma. ${ }^{46}$ Because of their location and rarity, noncutaneous melanomas often remain undetected until they reach an advanced stage, at which time treatment options are limited and outcomes are particularly poor. Evidence of the efficacy and safety of ipilimumab in patients with these rare melanoma subtypes is limited; however, preliminary data from expanded access programs (EAPs) or single-institution studies provide some indication of activity. ${ }^{47-51}$ Cumulatively, data have been reported from 228 patients with metastatic ocular melanoma treated with ipilimumab at $3 \mathrm{mg} / \mathrm{kg}$. Median OS ranged from 6 to 10.3 months, with one-third of patients surviving at least 1 year from starting treatment. Similarly, among approximately 200 patients with mucosal melanoma treated with ipilimumab $3 \mathrm{mg} / \mathrm{kg}$ within an EAP setting, 30\% to 40\% were alive 1 year after starting treatment. ${ }^{51}$

Another population of patients with a traditionally poor prognosis that may benefit from treatment with ipilimumab is that with brain metastases. Ipilimumab-activated $\mathrm{T}$ cells are thought to breach the blood-brain barrier, enabling them to mount an immune response in the brain. ${ }^{52}$ In a registrational phase III trial, 57 patients with asymptomatic brain metastases at baseline were treated with ipilimumab either alone or in combination with a gpl00 vaccine in the second-line setting. A consistent survival benefit was seen in each investigational group compared with the gpl00 comparator, with HRs of 0.76 and 0.70 , respectively, indicating a lower risk of death in ipilimumab-treated patients harboring brain metastases. ${ }^{53}$ Within EAPs, 860 patients with brain metastases have received ipilimumab $3 \mathrm{mg} / \mathrm{kg}$, with $19 \%$ to $25 \%$ of patients surviving at least 1 year. ${ }^{49,54}$ These results are encouraging, given the median OS for patients with brain metastases is 5 months after diagnosis, but this OS depends on the number and location of lesions (range 1-13 months). ${ }^{55}$

Preliminary data also suggest that vemurafenib and dabrafenib have activity in patients with melanoma that has metastasized to the brain. ${ }^{32,33,56-58}$ Patients with BRAF mutations are more likely than patients without the mutation to have central nervous system involvement at the time that they are diagnosed with distant metastatic disease. ${ }^{59}$ A phase II study of 172 patients with intracranial disease that assessed the response in patients treated with dabrafenib has been completed. In patients who received prior cranial-directed treatment, the response rate with dabrafenib was $31 \%$, which compared favor- ably with the cohort of patients who did not have prior therapy (response rate 39\%). ${ }^{60}$ Single-institution experience has shown that vemurafenib treatment in patients with BRAF-mutant melanoma with active brain metastases (18\% of patients in the retrospective study) resulted in an overall intracranial response rate of $40 \%$, which is higher than historical response rates to whole-brain radiotherapy ${ }^{61}$ In addition, an open-label singlearm study of vemurafenib in previously treated metastatic melanoma patients with brain metastases is currently ongoing. ${ }^{62}$ Though BRAF mutations are relatively common in cutaneous melanoma, they are extremely rare in areas with low exposure to sun and ultraviolet damage, as in ocular and mucosal melanoma. ${ }^{63-65}$ Researchers therefore assume that BRAF-inhibiting agents will have little value for these patient subpopulations. Many trials testing these agents have excluded patients with these melanoma histologies.

\section{- Applying Clinical Data to Practice}

According to the most recent NCCN guidelines, unresectable metastatic disease can be managed by systemic therapy, clinical trial, or best supportive care. ${ }^{44}$ Preferred systemic regimens are ipilimumab, vemurafenib, or dabrafenib (for patients with a documented BRAF mutation), treatment in a clinical trial, and high-dose IL-2. Thus, an important decision facing clinicians is which preferred regimen they should choose.

Ipilimumab and the enzyme inhibitors vemurafenib, dabrafenib, and trametinib have each demonstrated critical substantial patient benefits in the advanced melanoma population. On one hand, ipilimumab has been shown to reproducibly result in durable clinical benefit in the form of disease control (stable disease and objective responses) and long-term survival improvement, regardless of BRAF-mutation status, with approximately $20 \%$ of patients surviving at least 4 years. ${ }^{16,18,19,50}$ However, objective responses occur in less than 20\% of patients, and time is needed for clinical benefit to become apparent. Vemurafenib and dabrafenib, on the other hand, are associated with $40 \%$ to $50 \%$ response rates in patients with the BRAF mutation, and responses may be seen days to weeks after starting treatment. However, BRAF inhibitors are not suitable for the $50 \%$ to $60 \%$ of patients without a BRAF mutation; moreover, even in patients appropriate for BRAF-targeted therapy, the median duration of response is only 5 to 6 months. ${ }^{66}$ In the absence of prospective clinical data to guide the choice of treatment sequence in BRAF-mutated patients, many experts suggest treatment with a BRAF inhibitor first in patients with symptomatic, bulky, or rapidly growing disease. ${ }^{67-69}$ These patients are less likely to have time to build an effective immune response and benefit from immunotherapy and may achieve rapid improvement in symptoms with a selective BRAF inhibitor. Alternatively, in patients with asymptomatic or low-volume disease, immunomodulation can be considered, irrespective of the BRAF mutation status. The combination of 
BRAF- and MEK-inhibiting therapy appears to significantly improve PFS, possibly through delaying the onset of resistance in patients; however, this approach is investigational with confirmatory studies ongoing. ${ }^{36}$

Recent data suggest BRAF-resistance mechanisms select for a more aggressive disease, with around $50 \%$ of patients whose tumors fail BRAF-inhibitor treatment experiencing a very rapid evolution and progression of disease. ${ }^{68}$ In situations such as these, patients may be unable to complete therapy with the BRAF inhibitor and therefore benefit from subsequent immunotherapy. In patients with BRAF-mutated melanoma who have nonbulky, asymptomatic, or slowly progressing disease, first-line treatment with ipilimumab or high-dose IL-2 (in appropriate patients) could maximize the chance of long-term survival without compromising their ability to respond to subsequent BRAF inhibition. ${ }^{67-69}$ Furthermore, treatment with an MEK inhibitor in patients who have progressed on a BRAF inhibitor appears to provide minimal clinical activity. ${ }^{30}$

\section{Future Landscape}

The future of therapy for advanced melanoma most certainly lies in optimizing the sequencing and/or combinations of currently available therapies as well as incorporating new treatment options. Among the more promising agents are newer therapies that may provide patients with additional options. As previously mentioned, MEK inhibitors are likely to play a role in improving the response rate and delaying the onset of resistance in patients treated with BRAF inhibitors. ${ }^{27,28}$ Studies to date on the blockade of the programmed death 1 receptor (nivolumab; PD-1) and one of its ligands, PD-L1, have demonstrated impressive objective responses and durable tumor regression across a number of malignancies, including melanoma, while remaining safe and tolerable. ${ }^{70,71}$ These agents are currently being studied in combination with ipilimumab, and early phase results show that a majority of patients with advanced melanoma treated with ipilimumab and nivolumab have rapid and deep response of $80 \%$ or more tumor regression (NCT01783938; NCT01024231). ${ }^{72}$ Augmentation of the effects of ipilimumab are being investigated as well. One such promising approach includes the use of granulocyte-macrophage colony-stimulating factor (GM-CSF) with ipilimumab. In a study of 245 patients with advanced melanoma, patients were randomized to ipilimumab plus GM-CSF or ipilimumab alone. While response rates were similar, the investigation arm resulted in improved 1-year and overall survival. ${ }^{73}$ Studies are also exploring antigen-specific immunotherapy, such as talimogene laherparepvec, which has demonstrated a significant durable response rate and a trend toward improved OS in patients with advanced melanoma. ${ }^{74}$ Adoptive transfer of tumor-infiltrating lymphocytes or natural killer cells also appears to hold promise, but data to date are in highly selective patients. ${ }^{75,76}$ Continued research in these areas is being actively pursued.

\section{Limitations}

While notable advances have been made in the understanding and treatment of melanoma, it is important to recognize that the majority of patients with advanced disease will still die of the disease. The response rate for ipilimumab is only around $15 \%$, and long-term durability of response or stable disease occurs in a minority of patients. Kinase inhibitors targeting the RAS/RAF/MEK pathway are only applicable to 50\% of patients with specific mutations in the BRAF kinase, and only $50 \%$ of these patients respond to target-directed treatment. In patients responding to treatment, duration is typically short. Furthermore, rarer forms of the disease, such as acral lentiginous, mucosal, and uveal melanomas have other important features that have not been fully elucidated. For example, acral lentiginous and mucosal melanomas are much more likely to have activating mutations in c-KIT77 and may respond to agents such as imatinib. ${ }^{78}$ However, there are currently no FDA-approved therapies in melanoma for this particular mutation. Likewise, patients with ocular melanoma have not generally responded to currently available therapies, although MEK inhibitors hold some promise. ${ }^{79}$

\section{Conclusions}

Currently, the preferred treatment options for patients with advanced cutaneous melanoma comprise targeted therapy or immunotherapy. Targeted agents appear most useful for preventing early deaths among patients who urgently need a response in BRAF-positive disease, while immunotherapy is currently the only option that provides the proven possibility of long-term survival, regardless of BRAF status. Traditionally, the clinical value of cancer drugs has been determined by the median OS; however, this metric does not account for the fact that immunotherapies require time to maximize their antitumor activity, leading to durable responses and long disease-free or disease-stable intervals. Nor does the metric take into account the utility of providing patients with a rapid symptom-modifying response realized with targeted therapies. Immunomodulation effects persist in the absence of continued treatment; therefore, those patients living beyond the median survival have better survival chances, as evidenced by the plateau in survival curves and meaningful proportion of patients living 4 or 5 years from treatment. ${ }^{72}$ A substantial increase in life expectancy, reduction of tumor-induced symptoms, and durable disease control are valuable outcomes for patients with advanced melanoma, particularly if the patients have a wellpreserved quality of life with the capacity to remain productive members of society and/or spend quality time with their families. ${ }^{80}$ In a metastatic disease setting, payers should consider both long- and short-term outcomes and the potential impact of improved life expectancy and continued productivity on the true value of treatment. 
Author

ANTHONY J. OLSZANSKI, MD, RPh, is Director, Early Clinical Drug Development Phase 1 Trials Program, and Co-Director, Cutaneous Malignancies, Fox Chase Cancer Center, Philadelphia, Pennsylvania.

AUTHOR CORRESPONDENCE: Anthony J. Olszanski, MD, RPh, Director, Early Clinical Drug Development Phase 1 Trials Program, and Co-Director, Cutaneous Malignancies, Fox Chase Cancer Center, 333 Cottman Ave., Ste. C303, Philadelphia, PA 19111. Tel.: 215.214.1676; E-mail: Anthony.Olszanski@fccc.edu.

\section{DISCLOSURES}

Bristol-Myers Squibb provided support for this article. The author reports that he has served on advisory boards for Bristol-Myers Squibb and Genentech.

\section{REFERENCES}

1. American Cancer Society. Cancer facts \& figures 2014. Available at: http:// www.cancer.org/acs/groups/content/@research/documents/webcontent/ acspc-042151.pdf. Accessed March 7, 2014.

2. Bleyer A, O'Leary M, Barr R, Ries LAG, eds. Cancer Epidemiology in Older Adolescents and Young Adults 15 to 29 Years of Age, Including SEER Incidence and Survival:1975-2000. Bethesda, MD: National Cancer Institute; 2006.

3. Howlader N, Noone AM, Yu M, Cronin KA. Use of imputed populationbased cancer registry data as a method of accounting for missing information: application to estrogen receptor status for breast cancer. Am J Epidemiol. 2012;176(4):347-56.

4. Balch CM, Gershenwald JE, Soong SJ, et al. Final version of 2009 AJCC melanoma staging and classification. J Clin Oncol. 2009;27(36):6199-206.

5. Korn EL, Liu PY, Lee SJ, et al. Meta-analysis of phase II cooperative group trials in metastatic stage IV melanoma to determine progression-free and overall survival benchmarks for future phase II trials. J Clin Oncol. 2008;26(4):527-34.

6. Garbe C, Eigentler TK, Keilholz U, Hauschild A, Kirkwood JM. Systematic review of medical treatment in melanoma: current status and future prospects. Oncologist. 2011;16(1):5-24.

7. Mansfield AS, Markovic SN. Novel therapeutics for the treatment of metastatic melanoma. Future Oncol. 2009;5(4):543-57.

8. HYDREA (hydroxyurea) prescribing information. Bristol-Myers Squibb Co. Revised July 2011. Available at: http://packageinserts.bms.com/pi/ pi_hydrea.pdf. Accessed January 29, 2014.

9. Hodi FS, O'Day SJ, McDermott DF, et al. Improved survival with ipilimumab in patients with metastatic melanoma. N Engl J Med. 2010;363(8):711-23.

10. Robert C, Thomas L, Bondarenko I, et al. Ipilimumab plus dacarbazine for previously untreated metastatic melanoma. N Engl J Med. 2011;364(26):2517-26.

11. Chapman PB, Hauschild A, Robert C, et al., and BRIM-3 Study Group. Improved survival with vemurafenib in melanoma with BRAF V600E mutation. N Engl J Med. 2011;364(26):2507-16

12. Nadler E, Broderick WC, Zarotsky V, Kim J. How do medical and pharmacy directors perceive the value of new cancer drugs? Drug Benefit Trends. 2009;21:120-30.

13. Nadler E, Eckert B, Neumann PJ. Do oncologists believe new cancer drugs offer good value? Oncologist. 2006;11(2):90-95.

14. Yervoy (ipilimumab) prescribing Information. Bristol-Myers Squibb Co. Revised December 2013. Available at: http://packageinserts.bms.com/pi/ pi_yervoy.pdf. Accessed January 29, 2014.
15. Tarhini A, Lo E, Minor DR. Releasing the brake on the immune system ipilimumab in melanoma and other tumors. Cancer Biother Radiopharm. 2010;25(6):601-13.

16. Wolchok JD, Weber JS, Maio M, et al. Four-year survival rates for patients with metastatic melanoma who received ipilimumab in phase II clinical trials. Ann Oncol. 2013;24(8):2174-80.

17. Eggermont AM. Can immuno-oncology offer a truly pan-tumour approach to therapy? Ann Oncol. 2012;23(Suppl 8):viii53-57.

18. Prieto PA, Yang JC, Sherry RM, et al. CTLA-4 blockade with ipilimumab: long-term follow-up of 177 patients with metastatic melanoma. Clin Cancer Res. 2012;18(7):2039-47.

19. Lebbe C, Weber JS, Maio M, et al. Five-year survival rates for patients with metastatic melanoma treated with ipilimumab in phase II trials. Ann Oncol. 2012;23(Suppl 9):1116PD. [Abstract]

20. Wolchok JD, Hoos A, O'Day S, et al. Guidelines for the evaluation of immune therapy activity in solid tumors: immune-related response criteria. Clin Cancer Res. 2009;15(23):7412-20.

21. Hoos A, Eggermont AM, Janetzki S, et al. Improved endpoints for cancer immunotherapy trials. J Natl Cancer Inst. 2010;102(18):1388-97.

22. Ascierto PA, Kirkwood JM, Grob JJ, et al. The role of BRAF V600 mutation in melanoma. J Transl Med. 2012;10:85.

23. Chapman PB, Hauschild A, Robert C, et al. Updated overall survival results for BRIM-3, a phase III randomized, open-label, multicenter trial comparing BRAF inhibitor vemurafenib with dacarbazine in previously untreated patients with BRAF V600E-mutated melanoma. J Clin Oncol. 2012;30(Suppl 15):8502. [Abstract]

24. Sullivan RJ, Flaherty KT. Resistance to BRAF-targeted therapy in melanoma. Eur J Cancer. 2013;49(6):1297-304.

25. Fedorenko IV, Paraiso KH, Smalley KS. Acquired and intrinsic BRAF inhibitor resistance in BRAF V600E mutant melanoma. Biochem Pharmacol. 2011;82(3):201-09.

26. Trunzer K, Pavlick AC, Schuchter L, et al. Pharmacodynamic effects and mechanisms of resistance to vemurafenib in patients with metastatic melanoma. J Clin Oncol. 2013;31(14):1767-74

27. Flaherty KT, Infante JR, Daud A, et al. Combined BRAF and MEK inhibition in melanoma with BRAF V600 mutations. N Engl J Med. 2012;367(18):1694-703.

28. Falchook GS, Lewis KD, Infante JR, et al. Activity of the oral MEK inhibitor trametinib in patients with advanced melanoma: a phase 1 doseescalation trial. Lancet Oncol. 2012;13(8):782-89.

29. Hauschild A, Grob JJ, Demidov LV, et al. Dabrafenib in BRAF-mutated metastatic melanoma: a multicentre, open-label, phase 3 randomised controlled trial. Lancet. 2012;380(9839):358-65.

30. Kim KB, Kefford R, Pavlick AC, et al. Phase II study of the MEKl/MEK2 inhibitor trametinib in patients with metastatic BRAF-mutant cutaneous melanoma previously treated with or without a BRAF inhibitor. J Clin Oncol. 2013;31(4):482-89.

31. Das Thakur M, Salangsang F, Landman AS, et al. Modelling vemurafenib resistance in melanoma reveals a strategy to forestall drug resistance. Nature. 2013;494(7436):251-55.

32. Falchook GS, Long GV, Kurzrock R, et al. Dabrafenib in patients with melanoma, untreated brain metastases, and other solid tumours: a phase 1 dose-escalation trial. Lancet. 2012;379(9829):1893-901.

33. Trefzer U, Minor D, Ribas A, et al. BREAK-2: a phase IIA trial of the selective BRAF kinase inhibitor GSK2118436 in patients with BRAF mutation-positive (V600E/K) metastatic melanoma. Pigment Cell Melanoma Res. 2011;24:1020. [Abstract LBA1-1].

34. Klinac D, Gray ES, Millward M, Ziman M. Advances in personalized targeted treatment of metastatic melanoma and non-invasive tumor monitoring. Front Oncol. 2013;3:54. 
35. Wright CJ, McCormack PL. Trametinib: first global approval. Drugs. 2013;73(11):1245-54

36. Flaherty KT, Robert C, Hersey P, et al., for the METRIC Study Group. Improved survival with MEK inhibition in BRAF-mutated melanoma. N Engl J Med. 2012;367(2):107-14.

37. Weber JS, Kähler KC, Hauschild A. Management of immune-related adverse events and kinetics of response with ipilimumab. J Clin Oncol. 2012;30(21):2691-97.

38. Yervoy (ipilimumab): serious and fatal immune-mediated adverse reactions. Risk evaluation and mitigation strategy. Bristol-Myers Squibb. 2012. Available at: http://www.yervoy.com/hcp/rems.aspx. Accessed January 29, 2014.

39. Weber JS, Dummer R, de Pril V, Lebbé C, Hodi FS, for the MDX01020 Investigators. Patterns of onset and resolution of immune-related adverse events of special interest with ipilimumab: detailed safety analysis from a phase 3 trial in patients with advanced melanoma. Cancer. 2013;119(9):1675-82

40. Schadendorf D, Hodi FS, Robert C, et al. Pooled analysis of long-term survival data from phase II and phase III trials of ipilimumab in metastatic or locally advanced, unresectable melanoma. Paper presented at: 2013 European Society for Medical Oncology; September 27-October 1, 2013; Amsterdam, Netherlands. Abstract LBA24

41. Ribas A, Hodi FS, Callahan M, Konto C, Wolchok J. Hepatotoxicity with combination of vemurafenib and ipilimumab. N Engl J Med. 2013;368(14):1365-66

42. Infante JR, Fecher LA, Falchook GS, et al. Safety, pharmacokinetic, pharmacodynamic, and efficacy data for the oral MEK inhibitor trametinib: a phase 1 dose-escalation trial. Lancet Oncol. 2012;13(8):773-81.

43. Robert C, Schadendorf D, Messina M, Hodi FS, O'Day S, for the MDX010-20 investigators. Efficacy and safety of retreatment with ipilimumab in patients with pretreated advanced melanoma who progressed after initially achieving disease control. Clin Cancer Res. 2013;19(8):2232-39.

44. National Comprehensive Cancer Network website. NCCN Clinical Practice Guidelines in Oncology. Melanoma v2.2014. Available at: http:// www.nccn.org/professionals/physician_gls/pdf/melanoma.pdf. Accessed February 2, 2014

45. Swetter SM, Boldrick JC, Jung SY, Egbert BM, Harvell JD. Increasing incidence of lentigo maligna melanoma subtypes: northern California and national trends 1990-2000. J Invest Dermatol. 2005;125(4):685-91.

46. Tas F, Keskin S, Karadeniz A, et al. Noncutaneous melanoma have distinct features from each other and cutaneous melanoma. Oncology. 2011:81(5-6):353-58

47. Khan SA, Callahan M, Postow MA, et al. Ipilimumab in the treatment of uveal melanoma: the Memorial Sloan-Kettering Cancer Center experience. J Clin Oncol. 2012;30(Suppl 15):8549. [Abstract]

48. Khattak M, Fisher R, Hughes P, Gore M, Larkin J. Ipilimumab activity in advanced uveal melanoma. Melanoma Res. 2013;23(1):79-81

49. Lawrence D, McDermott D, Hamid O, et al. Treatment of patients with stage III or IV melanoma on an ipilimumab expanded access program (EAP): results for $3 \mathrm{mg} / \mathrm{kg}$ cohort. Presented at: Society for Melanoma Research 2012 Congress; November 8-11, 2012; Hollywood, CA.

50. Maio M, Bondarenko I, Robert C, et al. Four-year survival update for metastatic melanoma patients treated with ipilimumab plus dacarbazine in phase 3 study CA184-024. Ann Oncol. 2012;23(Suppl 9):367. [Abstract]

51. Del Vecchio M, Simeone E, Chiarion Sileni V, et al. Efficacy and safety of ipilimumab in patients with pretreated, mucosal melanoma: experience from Italian clinics participating in the European Expanded Access Programme (EAP). Ann Oncol. 2012;23(Suppl 9):1130P. [Abstract]

52. Engelhardt B, Coisne C. Fluids and barriers of the CNS establish immune privilege by confining immune surveillance to a two-walled castle moat surrounding the CNS castle. Fluids Barriers CNS. 2011;8(1):4.
53. Lebbé C, McDermott DF, Robert C, et al. Ipilimumab improves survival in previously treated, advanced melanoma patients with poor prognostic factors: subgroup analyses from a phase III trial. Ann Oncol. 2010;21(Suppl 8):13240. [Abstract]

54. Queirolo P, Simeone E, De Galitiis F, et al. Efficacy and safety data from patients with advanced melanoma and brain metastases participating in the European ipilimumab expanded access programme (EAP) in Italy. Poster presented at: European Society for Medical Oncology Congress; September 28-October 2, 2012; Vienna, Austria.

55. Eigentler TK, Figl A, Krex D, et al., on behalf of the Dermatologic Cooperative Oncology Group and the National Interdisciplinary Working Group on Melanoma. Number of metastases, serum lactate dehydrogenase level, and type of treatment are prognostic factors in patients with brain metastases of malignant melanoma. Cancer. 2011;117(8):1697-703.

56. Dummer R, Rinderknecht J, Goldinger SM, et al. An open-label pilot study of vermurafenib in previously treated metastatic melanoma patients with brain metastases. J Clin Oncol. 2011;29(Suppl 15):8548. [Abstract]

57. Kefford R, Arkenau H, Brown MP, et al. Phase I/II study of GSK2118436, a selective inhibitor of oncogenic mutant BRAF kinase, in patients with metastatic melanoma and other solid tumors. J Clin Oncol. 2010;28(Suppl 15):8503. [Abstract]

58. Long GV, Kefford RF, Carr PJA, et al. Phase 1/2 study of GSK2118436, a selective inhibitor of V600 mutant (mut) BRAF kinase: evidence of activity in melanoma brain metastases (mets). Poster presented at: European Society for Medical Oncology Congress; October 8-12, 2010; Milan, Italy. [Abstract LBA227]

59. Jakob JA, Bassett RL Jr, Ng CS, et al. NRAS mutation status is an independent prognostic factor in metastatic melanoma. Cancer 2012;118(16):4014-23.

60. Long GV, Trefzer U, Davies MA, et al. Dabrafenib in patients with Val600Glu or Val600Lys BRAF-mutant melanoma metastatic to the brain (BREAK-MB): a multicentre, open-label, phase 2 trial. Lancet Oncol. 2012;13(11):1087-109.

61. Harding JJ, Catalanotti F, Yaqubie A, et al. Vemurafenib in patients with BRAF-mutant melanoma with brain metastases. J Clin Oncol. 2013;31(Suppl 15):9060. [Abstract]

62. Dummer R, Goldinger SM, Turtschi CP, et al. Open-label pilot study of vemurafenib in previously treated metastatic melanoma ( $\mathrm{mM}$ ) patients (pts) with symptomatic brain metastases (BM). Poster presented at: European Society for Medical Oncology Congress; September 28-October 2, 2012; Vienna, Austria.

63. Maldonado JL, Fridlyand J, Patel H, et al. Determinants of BRAF mutations in primary melanomas. J Natl Cancer Inst. 2003;95(24):1878-90.

64. Wong CW, Fan YS, Chan TL, et al. BRAF and NRAS mutations are uncommon in melanomas arising in diverse internal organs. J Clin Pathol. 2005;58(6):640-44

65. Rimoldi D, Salvi S, Liénar D, et al. Lack of BRAF mutations in uveal melanoma. Cancer Res. 2003;63(18):5712-15.

66. Sosman JA, Kim KB, Schuchter L, et al. Survival in BRAF V600mutant advanced melanoma treated with vemurafenib. N Engl J Med. 2012;366(8):707-14.

67. Ascierto PA, Simeone E, Giannarelli D, Grimaldi AM, Romano A, Mozzillo N. Sequencing of BRAF inhibitors and ipilimumab in patients with metastatic melanoma: a possible algorithm for clinical use. J Transl Med. 2012:10:107

68. Ascierto PA, Simeone E, Grimaldi AM, et al. Do BRAF inhibitors select for populations with different disease progression kinetics? J Transl Med. 2013;11:61.

69. Jang S, Atkins MB. Which drug, and when, for patients with BRAFmutant melanoma? Lancet Oncol. 2013;14(2):e60-69. 
70. Topalian SL, Hodi FS, Brahmer JR, et al. Safety, activity, and immune correlates of anti-PD-1 antibody in cancer. N Engl J Med. 2012;366(26):2443-54.

71. Brahmer JR, Tykodi SS, Chow LQ, et al. Safety and activity of anti-PD-Ll antibody in patients with advanced cancer. N Engl J Med. 2012;366(26):2455-65

72. Wolchok JD, Kluger H, Callahan MK, et al. Nivolumab plus ipilimumab in advanced melanoma. N Engl J Med. 2013;369(2):122-33.

73. Hodi FS, Lee SJ, McDermott DF, et al., and Eastern Cooperative Oncology Group. Multicenter, randomized phase II trial of GM-CSF (GM) plus ipilimumab (ipi) versus ipi alone in metastatic melanoma: E1608. J Clin Oncol. 2013;31(Suppl 15):CRA9007. [Abstract]

74. Andtbacka RHI, Collichio FA, Amatruda T, et al. OPTiM: a randomized phase III trial of talimogene laherparepvec (T-VEC) versus subcutaneous (SC) granulocyte-macrophage colony-stimulating factor (GM-CSF) for the treatment (tx) of unresected stage IIIB/C and IV melanoma. J Clin Oncol. 2013;31(Suppl 15):LBA9008. [Abstract]
75. Rosenberg SA, Dudley ME. Adoptive cell therapy for the treatment of patients with metastatic melanoma. Curr Opin Immunol. 2009;21(2):233-40.

76. Parkhurst MR, Riley JP, Dudley ME, Rosenberg SA. Adoptive transfer of autologous natural killer cells leads to high levels of circulating natural killer cells but does not mediate tumor regression. Clin Cancer Res. 2011;17(19):6287-97.

77. Curtin JA, Busam K, Pinkel D, Bastian BC. Somatic activation of KIT in distinct subtypes of melanoma. J Clin Oncol. 2006;24(26):4340-46.

78. Carvajal RD, Antonescu CR, Wolchok JD, et al. KIT as a therapeutic target in metastatic melanoma. JAMA. 2011;305(22):2327-34.

79. Carvajal RD, Sosman JA, Quevedo F, et al. Phase II study of selumetinib versus temozolomide (TMZ) in gnaq/Gnall (Gq/1l) mutant (mut) uveal melanoma (UM). J Clin Oncol. 2013;31(Suppl 15):CRA9003. [Abstract]

80. Barzey V, Atkins MB, Garrison LP, Asukai Y, Kotapati S, Penrod JR. Ipilimumab in 2nd line treatment of patients with advanced melanoma: a cost-effectiveness study. J Med Econ. 2013;16(2):202-12. 